\title{
Automated method for determining the etch pits density on crystallographic planes of large semiconductor crystals
}

\author{
G.S. Pekar, A.A. Singaevsky, A.F. Singaevsky \\ V. Lashkaryov Institute of Semiconductor Physics, NAS of Ukraine, \\ 41, prospect Nauky, 03680 Kyiv, Ukraine, \\ E-mail: pekar@isp.kiev.ua,511alexsin@gmail.com
}

\begin{abstract}
A method for express automatic evaluation of the dislocation density on the crystal surfaces has been developed. The work involves creation of a software that allows automatical determining the number of etch pits with a defined geometric shape, which are seen in the microscope view field, and calculation of the density of those etch pits. In addition, adaptation of a metallographic microscope for the above measurements has been made. The developed method can be used to greatly speed up the maping of etch pits density over the area of large crystals. For example, duration of about 400 measurements of etch pits density made in various sites of $330 \times 150 \mathrm{~mm}$ surface of the optical germanium crystal plate and of maping the etch pits distribution over this surface made by the developed method is about $40 \mathrm{~min}$, while duration of the same measurements made by the traditional method for visual counting the number of etch pits seen in the eyepiece of the microscope is several dozens of hours. Use of the described method has allowed us to determine the geometric position of maximum internal stress in large optical germanium plates grown by horizontal unidirectional crystallization. This method has been already included in the metrological complex of serial production of large-area plates made of Na-doped optical germanium - a new material of infrared technique, developed and introduced into production at the V. Lashkaryov Institute of Semiconductor Physics, NAS of Ukraine.
\end{abstract}

Keywords: etch pits density, automation of measurements, crystal plate, optical germanium.

Manuscript received 11.12.15; revised version received 28.01.16; accepted for publication 16.03.16; published online 08.04.16.

\section{Introduction}

Estimation of the amount and type of dislocations in semiconductor crystals belongs to the most common methods for characterization of semiconductor materials [1]. Many experimental methods for determining the density of dislocation had been developed previously, and the most simple and universal of them is based on determination of the etch pits density on various crystallographic planes of the crystals [2]. Like many years ago, at present this determination is made visually, by counting the number of etch pits in the field of view of the optical microscope and performing an arithmetic division of the obtained number by the visible crystal area. These measurements are quite lengthy and laborintensive, but just they are commonly used both in 
research and factory laboratories, and in many higher educational institutions they are included in the appropriate laboratory workshops [3]. The disadvantages of this method are especially clearly evident when studying the distribution of dislocation density over the large-area crystal surfaces. The authors of this paper have encountered these difficulties in studying the mechanical stresses in large optical germanium plates (up to $600 \mathrm{~cm}^{2}$ in area) that are used as protective screens in modern thermal imaging systems. As was shown [4], for this purpose it is reasonable to evaluate distribution of the dislocation density over the surface of the plate, since the values of stress and dislocation density correlate with each other. However, to construct the maps of the dislocation density distribution over the surface of a large plate, one has to conduct microscopic measurements for many hours by counting the number of etch pits in the field of view in many regions of the plate surface [4]. In order to speed up and simplify these measurements, we have developed an express method, namely, developed a software that enables to get an image of the crystal surface and automatically determine the number of etch pits with the defined geometric shape in the microscope field of view as well as calculate the density of those etch pits. The used metallographic microscope was adapted to these measurements.

\section{Frame capture images in the metallographic microscope}

To determine automatically the density of etch pits, the metallographic microscope type MBI11U12 has been used. Instead of the eyepiece, a video camera with high resolution Sunkwang S2046 xaic/so was installed. A hardware solution was used to introduce a video stream and then to perform its processing, namely, the video controller type AverMEDIA-TV 402 with a function of fixing the frame was installed. As a result, one may obtain a streaming video of the crystal surface and to use it as an input to the developed program.

The used microscope has 4 modes of operation with magnifications $\times 500, \times 700, \times 1200$ and $\times 2500$. To improve the accuracy of determining the etch pits density, the mode of operation with a minimum magnification $\times 500$ was used. The crystal under investigation was placed on the object table. A special device allowed us to move the crystal plane up to $350 \times 160 \mathrm{~mm}$ in area along three coordinate axes, two of which (mutually perpendicular horizontal directions) lie in the plane of the plate, and one axis (vertical axis) is used for focusing.

The program provides an opportunity to determine the density of etch pits with various shapes, which is defined in the dialog. It makes the program rather universal.

The developed method was used to determine the etch pits density in crystals of some semiconductor materials, such as germanium, cadmium sulfide and cadmium selenide. This article details the results obtained in studies of optical germanium.

\section{Selection of the etchant for detecting the etch pits on the (111) surface of germanium crystals}

A lot of types of chemical etchants used to reveal the etch pits on different surfaces of germanium crystals may be found in scientific literature. We have discovered that the etch pits, most distinct in their shape, can be obtained on the (111) Ge surface by using the super oxoil etchant ( 1 part of $\mathrm{HF}+1$ part of $\mathrm{H}_{2} \mathrm{O}_{2}+$ 200 parts of $\mathrm{H}_{2} \mathrm{O}$ ) [2]. However, when using this etchant, duration of the etching process is very high, ranging from 16 to 24 hours. As shown by our experiments, this duration can be significantly reduced by increasing the contents of acid and hydrogen peroxide in the solution, and the best etchant can be prepared by 4-fold reduction in the water content, whereby the etching time can be shortened to $3 \ldots 4$ hours, without deterioration of the etch pit image.

\section{Software for automatic determination of the etch pits density}

\subsection{Functional diagram of the program}

The functional diagram of the program (after pressing the "Count") is shown in Fig. 1.

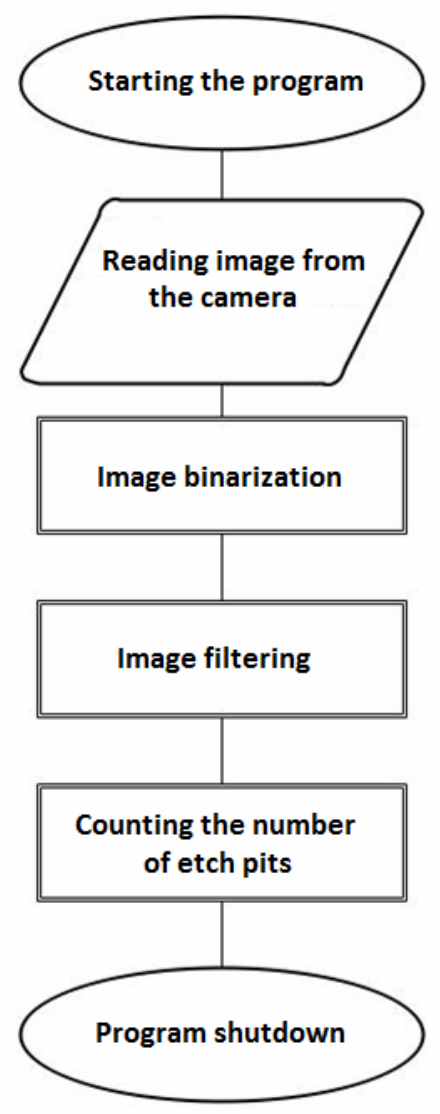

Fig. 1. Functional diagram of the program. 
After pressing the "Count", the program begins processing the selected image. Rgb2gray(); function converts the image into a white-gray-black. With the function $\operatorname{im} 2 \mathrm{bw}($,$) ; and a threshold intensity$ graythresh(); image binarization occurs (the image becomes black and white). Then the image filtering (noise processing) takes place, that is, screening out the areas which size doesn't exceed the adjusted number of pixels. Bwboundaries(,); function returns two arrays: one-dimensional array with the area number and the two-dimensional array of pixel coordinates on the boundary of each region.

Identification of the region shape occurs by the form of their morphometric features of the areas, such as:

"extent" - filling factor, that is the ratio of the object area to the area of rectangle that limits this object;

"solidity" - the coefficient of convexity, that is the ratio of the object area to the convex area of the object;

"eccentricity" - the eccentricity of an ellipse with principal inertia moments that are equal to the principal inertia moments of the object.

All parameters are represented by a number in the range $(0,1]$.

After finding objects (i.e., etch pits) that fall under the given parameters, their number and density are automatically calculated and displayed.
The accuracy of calculation and the degree of match between the real etch pits shape and the given shape of the objects are specified by the ideality factor, which varies in the range $(0,1]$ :

0 - calculation by the shape is not carried out,

1 -ideal shape is given.

It should be remembered that the etch pits shape is not perfect, and sometimes the etch pits may have an absolutely unexpected shape, so filtering by shape has the lowest priority.

Fig. 2 presents examples of the intermediate image filtering.

\subsection{Description of the developed program}

To automatically calculate the number of etch pits on the crystal surface, a program based on the application programs package for solving technical problems MatLab has been developed. In particular, the packages of Image Acquisition Toolbox and Image Processing Toolbox were used.

The program provides for the shoot of a video from a camcorder or loading of the extraneous image. To get started with the video camera, press the button "Start". In the first window will be the video that is recorded by the camcorder. To fix the studied area, click "Capture".

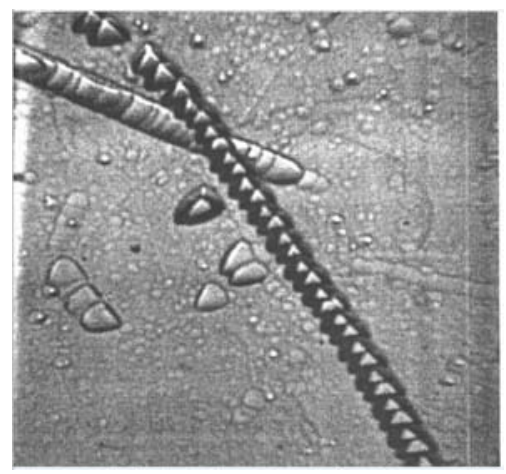

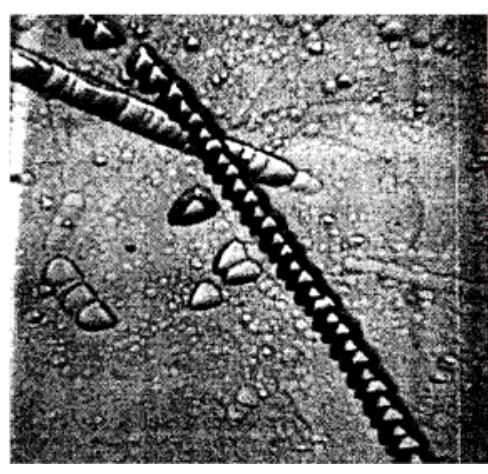

b

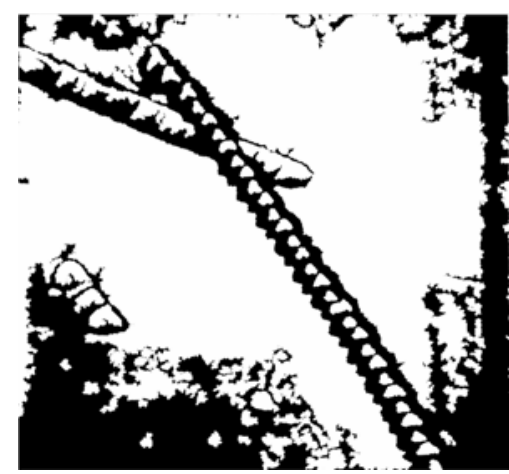

Fig. 2. The images of the optical germanium surface: (a) prior to computer processing, (b) a binary image, (c) after the noise processing.

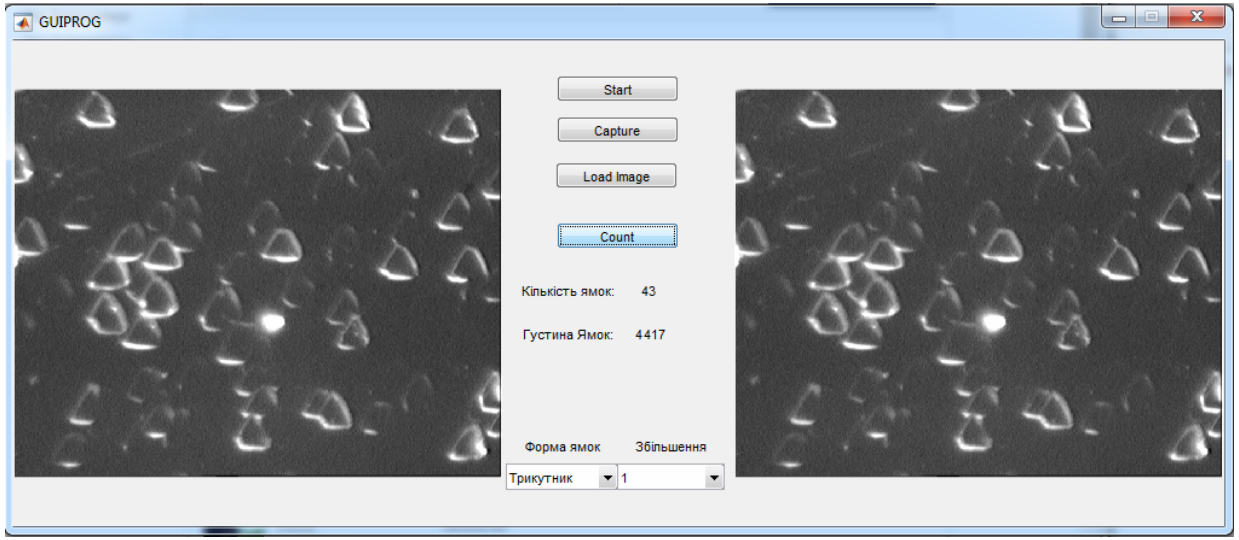

Fig. 3. An example of the work program. 


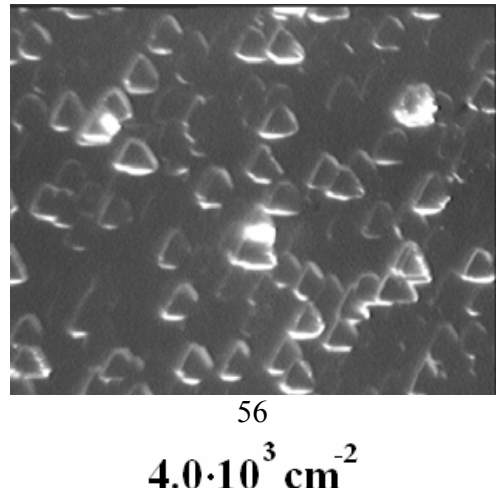

$4.0 \cdot 10^{3} \mathrm{~cm}^{-2}$

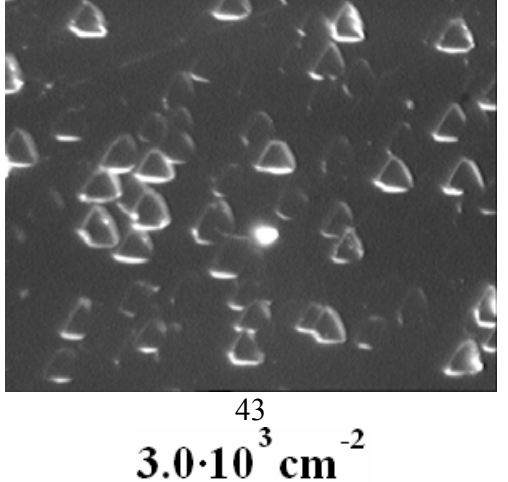

$3.0 \cdot 10^{3} \mathrm{~cm}^{-2}$

Fig. 4. Two examples of the obtained images. The numbers (higher values) and densities (lower values) of the etch pits calculated using the program are indicated.

If the user needs to examine the pre-recorded or extraneous images, press the button "Load". The studied area will appear in a second window. The user must choose the shape of the etch pits in the dialog box. Now, after pressing the "Count", in the output field "number of holes" will be a number that represents the number of etch pits in a given area. If the user sets the magnification of the microscope, in the region "Density pits" will be the number that represents the etch pits density in $\mathrm{cm}^{-2}$. Fig. 3.

An example of the work program is shown in

\section{Measurements}

As an example of the practical application of the developed method for rapid estimation of the etch pits density, adduced below are the results obtained in the study of (111) surface of optical germanium plate $330 \times 150 \mathrm{~mm}$ in size. Germanium was doped with $\mathrm{Na}$ during the crystal growing. The sodium concentration in the grown plate was about $2 \cdot 10^{14} \mathrm{~cm}^{-3}$. This material was developed by the authors previously $[5,6]$ and is currently being used for creating the various elements of infrared optics (lenses, screens, etc.). Before measurements, the plate surface was grinded and polished, and then it was chemically etched in accordance with the above etchant receipt and etching mode. Studies were performed on several randomly chosen areas in different parts of the plate surface.

Fig. 4 shows examples of the images obtained and the number and density of etch pits calculated by the program. Shown below are some examples of the obtained images, and the number and density of etch pits, which were calculated using the program. Note that the etch pits have a triangular shape on the investigated germanium crystal surface (111).

Using the method for statistical collection data, it has been found that the error of counting the etch pits density does not exceed 7\%. However, this error depends both on the correctness of program calibration and the quality of the initial image, so it may vary slightly both for the better and for the worse.

To determine the distribution of the etch pits density over the whole surface of the plate, we performed computerized calculations of the etch pits density in 384 regions over the surface of the crystal plate. Shown in Figs. 5 and 6 are the image of the investigated optical germanium plate and the dimensional diagram of the etch pits density based on the measurements described above, respectively. As seen, the value of etch pits density on the surface varied approximately from $6 \cdot 10^{2}$ to $5 \cdot 10^{3} \mathrm{~cm}^{-2}$. It is essential that duration of those experiments from the very beginning (i.e., from the crystal plate placement on the objective table of the microscope) to the end (i.e., to receiving the dimensional diagram) was about $40 \mathrm{~min}$. When using the conventional methods based on visual counting the same number of etch pits that can be seen in the eyepieces of the microscope, construction of such diagrams takes many hours.

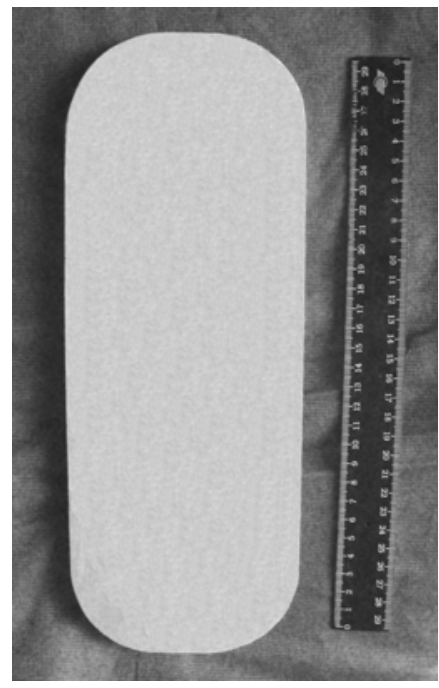

Fig. 5. Image of the optical germanium plate under investigation $330 \times 150 \times 20 \mathrm{~mm}$ in size. 


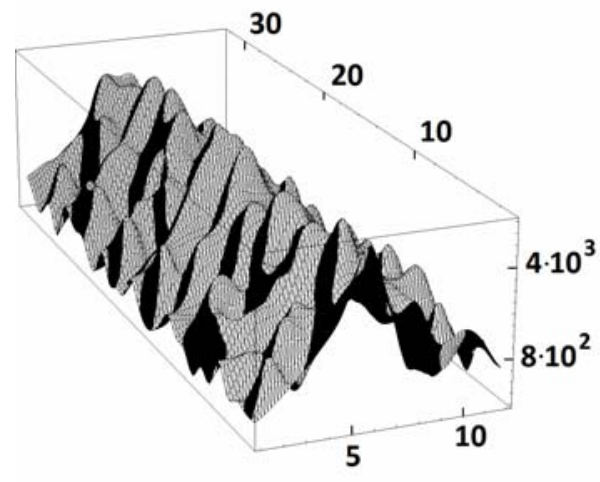

Fig. 6. The dimensional diagram of distribution of etch pits density over the surface of the optical germanium plate. The numbers along the horizontal axes indicate the geometric dimensions of plate in $\mathrm{cm}$, the numbers along the vertical axis - etching pits density in $\mathrm{cm}^{-2}$.

\section{Conclusion}

The method for express automatic evaluation of the dislocation density on the crystal surfaces (including large-area surfaces) with different crystallographic orientations has been developed. Created in this work is the software that enables to get the image of the crystal surface and automatically determine the number of etch pits with the defined geometric shape in the microscope field of view and to calculate the density of those etch pits. Adaptation of the metallographic microscope for the above measurements has been made. It includes the installation of a system for video fixation of the image as well as the installation of the table that allows to move the crystal (having the analyzed surface area up to $350 \times 150 \mathrm{~mm}$ ) in three coordinate directions relative to the microscope objective. The developed method was used for the rapid construction of maps of etch pits density distribution over the area of large crystals. Duration of 384 measurements in various plate regions, which were used to construct this map, did not exceed $40 \mathrm{~min}$. The developed method has been already included to the measuring complex of the mass production of large plates of Na-doped optical germanium - a new material of infrared technique, which was created and introduced into production at the V. Lashkaryov Institute of Semiconductor Physics, NAS of Ukraine.

\section{References}

1. E.Yu. Kokorish, N.N. Sheftal', Dislocations in semiconductor crystals // Sov. Phys. Usp. 3, p. 840849 (1961).

2. Etching of Semiconductors. Collection of papers. Formed by S.N. Gorin. Mir, Moscow, 1965 (in Russian).

3. Laboratory practical works on the course "Materials and components of electronic equipment”. Part 1: Educational handbook / V.I. Ratushny, A.Yu. Smolin, N.V. Litvin, N.V. Kapustin. National Research Nuclear University "Moscow Engineering Physics Institute", Moscow, 2012 (in Russian).

4. I.A. Kaplunov, Internal stresses and dislocation structure in germanium single crystals // Inorganic Materials, 42(6), p. 586-592 (2006).

5. G.S. Pekar, A.F. Singaevsky, Optical germanium // Patent of Ukraine for invention No. 81729, 25.01.2008, C30B 15/00, 29/08, 33/02 (in Ukrainian).

6. G.S. Pekar, A.F. Singaevsky, Na-doped optical Germanium bulk crystals // Appl. Phys. A, 108(3), p. 657-664 (2012). doi: 10.1007/s00339-012-6947-x. 